\title{
LA INCIDENCIA DE LAS TECNOLOGÍAS DE LA INFORMACIÓN Y LA COMUNICACIÓN EN EL DESARROLLO ORGANIZACIONAL DE LAS EMPRESAS DEL CANTÓN JIPIJAPA
}

\author{
AUTORES: Erick Geovanny Salazar Ponce ${ }^{1}$ \\ Zaine Bertyrene Rivadeneira Menéndez ${ }^{2}$ \\ Viviana Paola Castro López ${ }^{3}$
}

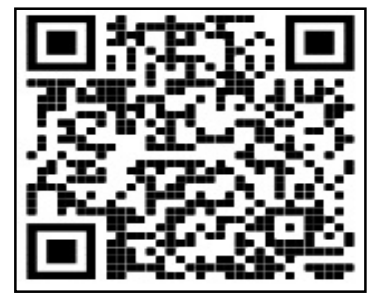

DIRECCIÓN PARA CORRESPONDENCIA: erick.salazar@unesum.edu.ec

Fecha de recepción: 29/08/2020

Fecha de aceptación: 28/11/2020

\section{RESUMEN}

Actualmente la innovación tecnológica es un eje importante dentro del desarrollo empresarial, con los cuales se adoptan cambios en los diferentes departamentos mejorando la ejecución en sus procesos cotidianos. Son múltiples los beneficios que aportan las Tecnologías de la Información y la Comunicación (TIC’s) en las diferentes áreas empresariales, siendo importante que toda entidad pública o privada se enfoque en cumplir sus metas. Es por ello, que se considera oportuno, determinar de qué manera la tecnología de la información y la comunicación incide en el desarrollo organizacional de las empresas del cantón Jipijapa. Para lo cual se aplicaron métodos de investigación como el exploratorio, analítico - sintético e inductivo - deductivo. Obteniendo como resultado que existe una mínima adquisición de estas herramientas en las empresas dedicadas a las actividades primarias y secundarias, siendo las actividades terciarias quienes han implementado en mayor medida equipos tecnológicos, por lo cual se han visto considerablemente potencializadas.

PALABRAS CLAVE: desarrollo organizacional, tecnologías de la información y comunicación, cultura, conocimiento, innovación, inestabilidad económica.

\section{THE INCIDENCE OF INFORMATION AND COMMUNICATION TECHNOLOGY IN THE ORGANIZATIONAL DEVELOPMENT OF THE JIPIJAPA CANTON COMPANIES}

\section{ABSTRACT}

\footnotetext{
${ }^{1}$ Doctor en Administración (PhD), Magister en Gerencia Educativa, Diplomado Superior en Gestión de Finanzas, Economista; Email: erick.salazar@unesum.edu.ec; código ORCID: 0000-0001-5732-5912

${ }^{2}$ Estudiante de la Universidad Estatal del Sur de Manabí; Email: zainerivadeneira@hotmail.com

${ }^{3}$ Ingeniera en Computación y Redes; Email: vivi.cas.lo@hotmail.com
} 
At the moment the technological innovation is an important axis within the business development, with which changes are adopted in the different departments improving the execution in their daily processes. There are multiple benefits that TIC'S bring in different business areas, being important that every public or private entity focus on meeting its goals. Therefore, it is considered appropriate to determine how information and communication technology affects the organizational development of companies in the Jipijapa canton. For which research methods such as exploratory, analytical - synthetic and inductive - deductive were applied. Obtaining as a result that there is a minimum acquisition of these tools in companies dedicated to primary and secondary activities, being tertiary activities who have implemented technological equipment to a greater extent, so they have been considerably enhanced.

KEYWORDS: organizational development, information and communication technologies, culture, knowledge, innovation, economic instability.

\section{INTRODUCCIÓN}

En la actualidad la sociedad del conocimiento por la que transitamos ha venido en un constante proceso de retroalimentación que provee un desarrollo plagado de cambios e inventos. Las tecnologías de la información y de la comunicación conocidas por su sigla TIC`S están asociadas a innovaciones comerciales, empresariales, sociales y legales.

Polanco (2006) la sociedad del conocimiento ya tiene instalado su paradigma: su bien más valioso es la inversión en capital intangible, humano y social, siendo sus activadores y factores clave el conocimiento y la creatividad; Kaufmann (2002) asevera que el mundo real y tangible se entrelaza con el virtual pasándose de la contigüidad a la conectividad, de las relaciones basadas en la proximidad física a la intermediación de ésta por sistemas tecnológicos de información y comunicación.

En Ecuador en el año 2015 el Instituto Nacional de Estadística y Censos (INEC) realizó una encuesta industrial sobre Tecnologías de la Información y Comunicación (TIC) a 3.245 entre micros, pequeñas, medianas y grandes empresas dedicadas a manufactura, minería, comercio y servicios, donde se muestra que, el $67 \%$ de las empresas invierten en TIC, siendo las microempresas las que tienen la menor inversión, con un total de 57 microempresas encuestadas tan solo 8 han optado por invertir en TIC, situación que demuestra el alto grado de desinterés de las organizaciones en incluir o mejorar equipos tecnológicos, incurriendo en problemas de ineficiente comunicación entre las áreas y el personal.

También se registró que aproximadamente tres microempresas utilizan aplicaciones para el procesamiento de información tipo ERP (Enterprise Resource Planning - Planificación de Recursos Empresariales) que de acuerdo al autor (Cabello, 2018) los ERP son sistemas encargados de integrar la gestión de los diferentes departamentos de la empresa, unificando y centralizando todos los datos que se desee, sin importar la distancia física, siendo estas operaciones las piezas claves pero mínimamente utilizadas por las compañías para lograr un desarrollo organizacional. Aunque se considera imprescindible contar con expertos en TIC dentro de las organizaciones para erradicar el analfabetismo digital de los colaboradores de la misma, 
únicamente 1.263 es decir el 38,92\% de las empresas cuentan con estos especialistas dentro de su estructura.

Las herramientas de comunicación también contribuyen a la optimización de recursos económicos que benefician el comercio y adquisición de insumos tecnológicos que influyen de manera positiva en la empresa. Es importante recalcar que las pymes pueden presentar dificultades administrativas al momento de implementar software relacionado a la interacción con proveedores o consumidores potenciales, ya que, al no tener talento humano idóneo en el área, estos grupos deberían someterse a capacitaciones intensivas en beneficio de la empresa.

\section{Las TIC en la dirección y la gestión de recursos humanos}

En la década de los noventa se redescubre que lo más importante de la empresa no son sus recursos materiales, sino sus personas, dotadas de conocimientos, creatividad, iniciativa. Se habla cada vez más de las empresas basadas en el conocimiento (Escorsa Castells \& Valls Pasola, 2003). Poseer activos tangibles no aporta beneficios a las empresas si estas no son utilizadas de forma correcta por los operadores para poder aprovechar al máximo las ventajas que tiene la incorporación de los equipos, situación que es importante que la alta gerencia tome en cuenta cuando desee incorporar nueva tecnología dentro de los procesos organizacionales.

La incursión de las Tecnologías de la Información y las Comunicaciones (TIC) en el contexto organizacional ha revolucionado el pensamiento administrativo y gerencial, esto se evidencia en varios aspectos, uno de ellos es la forma como se gestiona el talento humano (Riascos Erazo \& Aguilera Castro, 2011). Las TIC se han convertido en la opción más viable para él área administrativa de las empresas que, aunque estas en ciertas ocasiones amerita una alta inversión los benéficos que se obtienen son importantes como mejorar la comunicación entre las áreas, departamentos y trabajadores, compartir información al instante minimizando el tiempo de los procesos teniendo resultados óptimos mientras se reduce el uso de recursos con los que cuenta la organización.

Esto ha supuesto un reto en la dirección y gestión de los RRHH, que cada vez se preocupa más por desarrollar estrategias de comunicación y de motivación encaminadas a hacer que los trabajadores compartan la visión y misión de la empresa. Las TIC ofrecen a los departamentos o encargados de RRHH herramientas de gestión que facilitan y mejoran enormemente su trabajo, así como plataformas de aprendizaje a distancia que favorecen el acceso a la formación y, con ello, contribuyen a una mayor eficiencia de los trabajadores y, consecuentemente, de la empresa (Promove Consultoria e Información SLNE, 2012).

Las tecnologías de la información y comunicación aportan múltiples opciones de preparación continua para el recurso humano con el que cuentan las empresas, siendo esto necesario para alcanzar el máximo interés de los colaboradores con el fin de vincularlos voluntariamente al complimiento de los objetivos y metas empresariales obteniendo resultados eficientes y perdurable. 
La transformación del trabajo que realizan las personas en una organización, es una de las principales manifestaciones del proceso de cambio que se produce en el tránsito de una economía industrial a una economía global fundamentada en la gestión del conocimiento (Pérez Llana, Pascual, Cueto, \& Carlón, 2012). Adaptarse a las necesidades de la sociedad y competir con las demás empresas requiere cambios progresivos dentro de las áreas organizacionales, donde se debe considerar como principal elemento de transformación al talento humano para lograr pasar de acciones automáticas a operaciones basadas en conocimiento.

\section{Herramientas Informáticas para la Gestión del Conocimiento}

La Gestión del Conocimiento se refiere a gestión de los activos intangibles que generan valor para una organización y el aprendizaje organizacional es su principal herramienta (González \& Filippi Sánchez, 2010). En la actualidad, mantener un ambiente de instrucción organizacional continua aporta fortalezas empresariales y una constante actualización de los conocimientos de quienes son parte de la estructura corporativa sin importar el rango en el que se encuentren pudiendo ser desde el escalón más bajo hasta el más alto de la jerarquía, aportando conjuntamente al desarrollo integral de la empresa.

La gestión del conocimiento es un modelo de gestión organizacional, que fomenta la creación de una cultura organizacional dentro de un entorno de colaboración que fomente la cooperación interpersonal y estimule el aprendizaje colaborativo. Además la gestión del conocimiento, busca generar las condiciones necesarias para que la información fluya, de forma oportuna en toda la organización, sobre la base de un necesario soporte tecnológico que facilite y agilice este flujo de la información y de conocimiento, de modo que con ellos se facilite la toma de decisiones, en función del cumplimiento de los objetivos de la organización. (Quintanilla Juárez, 2014)

Las tecnologías de la información y comunicación son instrumentos de apoyo en la gestión del conocimiento ya que permiten el flujo de información de forma vertiginosa optimizando tiempo, mejorando la comunicación entre áreas de la organización permitiendo la colaboración adecuada de los participantes que son necesarios e imprescindibles dentro de cada proceso que lleve a cabo la empresa y que de forma indirecta y paulatina se convierta para los trabajadores en hábitos positivos para así lograr una cultura organizacional que disminuya el miedo y la resistencia a cambios estratégicos necesarios para lograr un desarrollo organizacional incesante.

\section{Desarrollo organizacional}

Frente a los constantes cambios económicos, tecnológicos, sociales y culturales las empresas se ven en la necesidad de adoptar nuevas reglas, nuevos equipos, nuevas creencias e incluso nuevos trabajadores que compartan la visión organizacional.

Muchas organizaciones modernas han logrado integrar los cambios tecnológicos y de información; en cambio, muchas otras no han conseguido ajustarse y asimilar el cambio social y cultural debido a su incapacidad de adaptación e integración del cambio tecnológico. En realidad, así como sucedió con el uso de la computadora, en ocasiones, el atraso cultural frena el uso adecuado de las nuevas tecnologías (Guízar Montúfar, 2013). 
La constante innovación de la tecnología y las nuevas técnicas informativas y de comunicación han resultado beneficioso para aquellas empresas que con anterioridad se venían preparando con conocimientos, destrezas y el mejoramiento de las habilidades individuales mediante la práctica, capacitaciones que en forma conjunta lo que crea confianza y la capacidad de avanzar culturalmente logrando de forma óptima un desarrollo organizacional.

El Desarrollo Organizacional ayuda a los líderes a abordar y adoptar el cambio desde la perspectiva de que el cambio es una oportunidad y no una amenaza. (Teran, Cañizales, Infante, \& Albornoz, 2002). Realizar cambios estratégicos en las relaciones interpersonales, sus funciones y la efectividad de los mismos es parte de lograr el desarrollo organizacional que las empresas necesitan para la mejora continua de la misma.

Para (Maldonado, 2017) de acuerdo a su propia definición presupone características como:

- Orientación sistémica.

- Agente de cambio.

- Procesos grupales.

- Desarrollo de equipos.

- Retroalimentación.

Lo esencial en el desarrollo organizacional es vincular a todo el equipo humano en el progreso de la empresa, acción que puede ser posible si se conoce con precisión el estado actual de la organización para así lograr mejorar la salud de la misma, es decir conocer y definir los problemas existentes de cualquier índole en departamentos o del personal de forma individual pero que repercuten en el accionar eficiente de otras áreas o actividades y así formular soluciones pertinentes que imposibiliten el estancamiento de la empresa.

En cuanto a los elementos de la cultura organizacional, los mismos están dados por los caracteres del entorno compartidos; tecnología, hábitos y modos de conducta; cargos y funciones; roles; ritos, ceremonias y rutinas; redes de comunicación; sistema de valores, mitos y creencias. (Universidad Nacional de la Plata, 2007). Es importante lograr una adaptación en conjunto de todos los que integran la empresa, puesto que se obtendrán mejores relaciones interpersonales, colaboración entre áreas, mejora en la comunicación, obtención de información de forma instantánea, apoyo para el entendimiento y acoplamiento a los cambios culturales aplicados a nivel organizacional.

\section{DESARROLLO}

Se consideró la aplicación del método exploratorio para la obtención de datos acerca de las empresas que se encuentran registradas en el Servicio de Rentas Internas (SRI) zona 4, distrito Jipijapa en el periodo fiscal 2018 hasta marzo 2019, con sus respectivas actividades comerciales. Por medio del método inductivo, se determinó el tipo de encuestas que se van a realizar a las 
empresas del cantón Jipijapa, también fue oportuno para establecer las particularidades y beneficios que vinculan las pymes con el uso de la tecnología de la información y la comunicación. En cuanto se refiere al método deductivo podemos vincularlo al desarrollo diferenciado que tienen las empresas que utilizan la tecnología de la información y la comunicación para agilitar procesos administrativos, económicos y productivos.

Una vez realizada la encuesta a las empresas del cantón Jipijapa, hemos utilizado el método analítico el cual nos dice, que para un mejor entendimiento de nuestro caso debemos descomponer los objetos de estudios, luego analizar de manera individual cada una de sus variables, siendo esta actividad primordial para el desarrollo y síntesis de las conclusiones.

\section{RESULTADOS}

Ante los múltiples beneficios que aportan las tecnologías de la información y la comunicación en el desarrollo organizacional de las empresas, se consideró oportuno enlistar algunos de ellos, los cuales han sido agrupados de acuerdo a la orientación que se le dé a cada uno de los equipos tecnológicos adquiridos.

Tabla 1 Beneficios del uso de las TIC'S en el Desarrollo Organizacional

\begin{tabular}{|c|c|}
\hline $\begin{array}{l}\text { Equipos tecnológicos orientados a la } \\
\text { información }\end{array}$ & $\begin{array}{l}\text { Equipos tecnológicos orientados a la } \\
\text { comunicación }\end{array}$ \\
\hline 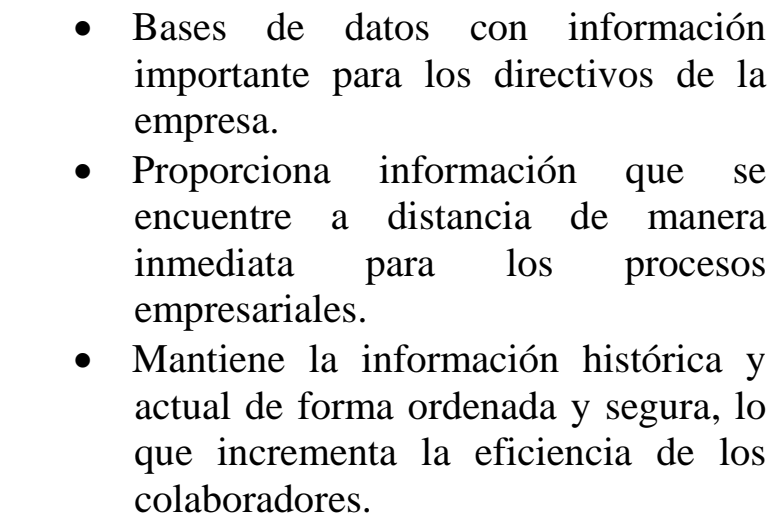 & $\begin{array}{l}\text { - Vinculación entre la dirección y } \\
\text { trabajadores que no se encuentren } \\
\text { en el mismo lugar físico. } \\
\text { - Facilita la correcta dirección de } \\
\text { los mensajes sin alterar su } \\
\text { contexto. } \\
\text { - Mejora las conductas } \\
\text { interpersonales de quienes son } \\
\text { parte de la organización, logrando } \\
\text { un correcto clima }\end{array}$ \\
\hline $\begin{array}{l}\text { - Se omite toda información que se } \\
\text { encuentra duplicada conservando la } \\
\text { original evitando datos que alteren los } \\
\text { resultados de los procedimientos } \\
\text { administrativos. } \\
\text { - Genera de forma automática informes } \\
\text { con información registrada } \\
\text { previamente, optimizando el tiempo y } \\
\text { aumentando la productividad de los } \\
\text { trabajadores. } \\
\text { Búsquedas inteligentes en las bases de } \\
\text { datos mediante palabras claves, } \\
\text { reduciendo el tiempo en la toma de }\end{array}$ & $\begin{array}{l}\text { Organizacional } \\
\text { - Maximiza la participación de los } \\
\text { trabajadores en el cumplimiento } \\
\text { de las metas planteadas. } \\
\text { - Crea un ambiente laboral } \\
\text { confiable que conlleva a una } \\
\text { mejor adaptación a los cambios } \\
\text { establecidos. } \\
\text { - Genera la integración de todos los } \\
\text { trabajadores en el cumplimiento } \\
\text { de los objetivos, acción } \\
\text { fundamental en el desarrollo } \\
\text { organizacional. }\end{array}$ \\
\hline
\end{tabular}

166 UNESUM-Ciencias. Publicación cuatrimestral. Vol. 4, No. 4 (Septiembre-Diciembre), Año 2020. 


\begin{tabular}{|l|ll|}
\hline decisiones. & $\bullet$ & $\begin{array}{l}\text { Profundiza el conocimiento de las } \\
\text { reglas, procedimientos y políticas. }\end{array}$ \\
- Optimización de recursos tangibles. & Facilita la colaboración de las \\
personas & & \\
\hline
\end{tabular}

Elaborado por: Investigadores

En el cantón Jipijapa, el 73\% de los administradores de las empresas desconocen los beneficios que traen consigo implementar tecnologías de la información en el desarrollo organizacional de las empresas, dejando como resultado una mínima cantidad de directivos que si conocen acerca de las ventajas que aporta la adquisición de equipos tecnológicos orientados a un adecuado manejo de la información, así como de la importancia de mantener a cada uno de los departamentos y personal internamente comunicados.

Este desconocimiento se ve reflejado en el bajo nivel de inversión que ha realizado el $75 \%$ de las empresas en cuanto a tecnologías de la información y comunicación en el año 2018, cifra que contrasta considerablemente con el 9\% correspondiente a aquellas que si han decido invertir en equipos tecnológicos en pro del desarrollo organizacional.

El 95\% de aquellas personas que toman las dediciones dentro de las empresas, han considerado como mejor opción la generación de conocimientos empíricos, los cuales se obtienen a través de la experiencia que se gana con la implementación en estos casos de equipos tecnológicos, esta acción puede beneficiar en cierta medida a la empresa, sin embargo, las ventajas podrían verse reflejadas a largo plazo, ya que los operarios no poseen el conocimiento necesario para la utilización de estas herramientas, corriendo el riesgo de que estas cumplan su tiempo de vida útil antes de alcanzar su máximo potencial, mientras que otras pocas, el $4 \%$ han preferido brindar capacitaciones a su personal sobre tecnología, siendo tan solo el 1\% de quienes están al frente de estas organizaciones los que han decidido incorporar a su estructura laboral a personal especializado en tecnologías de la información y comunicación, que imparta nuevos conocimientos tecnológicos y genere un cambio en la cultura organizacional.

Tabla 2 Actividades para desarrollar o mejorar conocimientos sobre TIC'S

\begin{tabular}{|l|l|l|}
\hline Opciones & Frecuencia & Porcentaje \\
\hline Capacitación sobre tecnología & 16 & $4 \%$ \\
\hline Personal especializado en TIC & 2 & $1 \%$ \\
\hline Implementar equipos tecnológicos (práctica) & 338 & $95 \%$ \\
\hline Total & $\mathbf{3 5 6}$ & $\mathbf{1 0 0 \%}$ \\
\hline \multicolumn{2}{|c|}{$\begin{array}{c}\text { Fuente: Empresas del cantón Jipijapa } \\
\text { Elaborado por: Investigadores }\end{array}$}
\end{tabular}

En la zona urbana y rural del cantón Jipijapa, el 90\% de las empresas son consideradas como microempresas por contar de 1 a 9 personas. De estas, la gran mayoría se enfrentan a una alta 
competencia en cada uno de los diferentes mercados a los que están inmersos, lo que amerita que deban tomar medidas para disminuir este impacto, sin embargo, en la mayoría de las empresas del cantón tan solo el $10 \%$ de los empleados utilizan equipos tecnológicos adecuados para la realización de sus actividades, lo que deja en evidencia, que las organizaciones a nivel local aun realizan la mayor cantidad de sus procesos o trabajos de forma manual, viéndose involucrado el tiempo que los trabajadores necesitan para ejecutar de forma correcta cada una de sus actividades, siendo la comunicación interna la actividad que se realiza con mayor frecuencia utilizando equipos tecnológicos como ordenadores, telefonía móvil e internet, seguido actividades de marketing, financieras y administrativas, como se detalla en la tabla 3.

Tabla 3. Equipos tecnológicos utilizados por las empresas

\begin{tabular}{|l|l|l|l|l|}
\hline Opciones & Administración & Finanzas & Comunicación interna & Marketing \\
\hline Internet & $13 \%$ & $16 \%$ & $61 \%$ & $55 \%$ \\
\hline Ordenadores & $21 \%$ & $24 \%$ & $6 \%$ & $29 \%$ \\
\hline Telefonía móvil & $12 \%$ & $8 \%$ & $54 \%$ & $22 \%$ \\
\hline Telefonía fija & $4 \%$ & $0 \%$ & $2 \%$ & $0 \%$ \\
\hline Correo electrónico & $7 \%$ & $10 \%$ & $9 \%$ & $1 \%$ \\
\hline Word, Excel, PowerPoint & $3 \%$ & $29 \%$ & $0 \%$ & $0 \%$ \\
\hline \multicolumn{5}{|r}{ Fuente: Empresas del cantón Jipijapa } \\
Elaborado por: Investigadores
\end{tabular}

La poca utilización de tecnologías de la información y comunicación en el área administrativa de acuerdo al 45\% de los emprendedores locales, se debe al desconocimiento de los beneficios que estos equipos aportan al desarrollo organizacional de las empresas, a su vez el $30 \%$ considera que el alto costo que tienen estas herramientas es un factor determinante al momento de adquirir equipos tecnológicos, mientras que el 15\% afirma que la falta de información acerca del correcto uso de las TIC'S es aquel factor que impide su implementación en las tareas administrativas que se llevan a cabo en las organizaciones.

Es imprescindible contar con información actualiza de cada una de las áreas con las que cuenta la empresa, esto se debe a que cada acción que se realice va a influir positiva o negativamente si no se tiene presente todos los aspectos importantes de los que dependen las demás actividades para llevar a cabo un proceso, ante esto, y considerando al bajo porcentaje de empresas que han invertido considerablemente en tecnologías de la información y comunicación se ha notado una mejora en la mayoría de las actividades administrativas, como la organización que se ha visto una mejoría en el $36 \%$ de las empresas, ya que les permite plantear un orden de las acciones a realizar, identificar y brindar las herramientas necesarias, seguida del control que realiza el administrador, inspeccionando y evaluando que los trabajos se realicen de acuerdo a las políticas, posteriormente ha mejorado la coordinación, permitiéndoles vincular cada una de las tareas generando un mejor resultado de las mismas, la planeación con una mejoría en el $14 \%$ de las empresas la cuales sirve de guía para el cumplimiento de los objetivos mediante la creación de estrategias adecuadas, siendo la dirección la actividad menos empleada con las TIC’S con un 9\%. 
Tabla 4. Actividades administrativas que han mejorado con el uso de TIC'S

\begin{tabular}{|l|l|l|}
\hline Opciones & Frecuencia & Porcentaje \\
\hline Planeación & 49 & $14 \%$ \\
\hline Organización & 129 & $36 \%$ \\
\hline Dirección & 32 & $9 \%$ \\
\hline Coordinación & 65 & $18 \%$ \\
\hline Control & 81 & $23 \%$ \\
\hline Total & $\mathbf{3 5 6}$ & $\mathbf{1 0 0 \%}$ \\
\hline
\end{tabular}

Fuente: Empresas del cantón Jipijapa

Elaborado por: Investigadores

A pesar de que el $97 \%$ de los directivos consideran que es muy importante digitalizar la información de las empresas, es muy reducido el número de organizaciones que hacen uso de bases de datos, donde se incluyan datos acerca de los equipos y materiales de oficina, trabajadores y producción disponible, siendo la información acerca de los ingresos y egresos los que son registrados en mayor medida por quienes están al mando de estas, cabe recalcar que esta medida es empleada solo por el $29 \%$ de empresas, considerando como razón principal que en el cantón Jipijapa la mayoría de empresas se encuentran constituida por familias, las cuales no consideran necesario registrar información de forma ordenada y segura, ni llevar un control de las actividades que se ejecuten dentro del negocio por sentir la seguridad de que sus familiares no harán ningún daño a la organización.

Aquellas empresas que se dedican a la comercialización de productos u ofrecer servicios que requieran de otras empresas proveedoras, suelen llevar un registro que se elabora de forma manual acerca de los productos que reciben por parte de estas, sin embargo, estos registros al encontrarse de forma física con el paso del tiempo no podrían ser comparados ya que están expuestos a diversos daños o pueden ser extraviados. Considerando a las empresas que en cierta medida han invertido en tecnologías de la información y comunicación, el 92\% pudo percibir una mejora considerable en el tiempo de respuesta que la organización tiene con sus clientes, mejorando la atención, creando fiabilidad y atrayendo nuevos consumidores, acciones que maximizan los ingresos.

Los equipos tecnológicos que fomentan la comunicación, han generado que exista en el $87 \%$ de empresas una buena relación entre la organización y los proveedores, donde prime una correcta emisión y recepción de cualquier tipo de información, de igual forma con los trabajadores de los negocios en un $81 \%$, donde adicionalmente se crea un ambiente laboral favorable para la ejecución de cada una de las tareas que se realizan de forma individual en cada uno de los departamentos, haciendo que estos cumplan con sus procesos de forma eficiente, aportando favorablemente al desarrollo de las actividades administrativas de las empresas del cantón.

A su vez las herramientas tecnológicas orientadas a la información que son aplicadas por ciertas organizaciones jipijapenses, han permitido que la información circule de forma segura y esté al

(c) Universidad Estatal del Sur de Manabí. Jipijapa, Ecuador. 
alcance del personal que la necesite con datos actuales, permitiéndoles tener una visión más amplia y confiable de lo que ocurre en el presente de la organización, información que le servirá en el futuro para enfocarse y trabajar en el fortalecimiento de las debilidades que presente la empresa.

Tabla 5. Empresas que se ha potencializado con el uso de TIC'S

\begin{tabular}{|c|c|c|}
\hline Primaria & Secundaria & Terciaria \\
\hline Avícola Orlando & Aqua Santa & Compañía de Taxis \\
& & Ejecutivo Vencedores \\
& & S.A. Transejevens \\
& & - Oceanside FarmKitchen \\
& & - JipiTv \\
& & - Satori \\
& & - RIVSU \\
& & \\
\hline
\end{tabular}

Elaborado por: Investigadores

Como se muestra en la tabla 5, es evidente que en el cantón Jipijapa existen un reducido número de administradores que han optado por implementar equipos tecnológicos vinculados a la información y comunicación, por ello las empresas que se han visto potencializadas con el uso de las tecnologías de la información y comunicación son mínimas, las cuales fueron agrupadas de acuerdo a al tipo de actividades económica que realizan cada una de ellas.

\section{DISCUSIÓN}

Se obtuvieron datos que no han sido encontrados previamente, como el porcentaje de trabajadores que utilizan equipos tecnológicos para la realización de sus actividades, la cantidad de administradores que tienen conocimiento acerca de los beneficios que se obtienen en el desarrollo organizacional de las empresas por el uso de tecnologías de la información y comunicación, así como el nivel de inversión en TIC’S que han realizado las empresas.

Existieron limitaciones en la elaboración del trabajo de campo, es decir al realizar las encuestas a las empresas, en las cuales en una que otras de estas no se encontraban los administradores de las mismas, por lo que se optó por realizar el levantamiento de información a quienes nos atendían, siendo en ciertas ocasiones familiares como su esposo/a, hijos/as, o algún otro colaborador.

A partir de los resultados que han sido expresados anteriormente, se confirma la hipótesis general, planteada en relación a la incidencia que tienen las tecnologías de la información y comunicación en el desarrollo organizacional de las empresas del cantón Jipijapa, ya que quienes han realizado altas e incluso medianas y bajas inversiones en estas herramientas tecnológicas, han tenido un mejoramiento en el rendimiento de los trabajadores, (ver anexo, tabla 13), siendo esto indispensable para el complimiento de las tareas, adaptación a los cambios, entre otras, que logran en el trabajador satisfacción por su trabajo. 
En el año 2018, el autor Cano Pita Galo, en su investigación titulada "Las TICS en las empresas: evolución de la tecnología y cambio estructural en las organizaciones” obtuvo como resultado lo siguiente. Vemos entonces como la variable tecnología siempre está presente en cada etapa del desarrollo de la estructura empresarial, por lo cual se podría concluir una relación muy estrecha entre evolución y tecnología. Con base a los estudios de algunos investigadores del área revisados en la presente investigación, se puede inferir de manera concluyente la relación directa que existe entre la evolución y cambio estructural en las organizaciones, producto de la adopción por parte de las mismas, de nuevas tecnologías de información y comunicación, adoptadas inicialmente como herramientas para afrontar nuevas realidades del entorno, lo cual produjo posteriormente en ellas cambios profundos en su diseño estructural, cultura y clima organizacional (Cano Pita, 2018).

Es notable la escasa estructura tecnológica que poseen las empresas del cantón Jipijapa, debido a que no existe una cultura para implementar estas herramientas en la realización de las actividades diarias y pese a la alta competencia que presentan no se muestra por parte de los administradores mayor interés en innovar los equipos o hacer uso de aplicaciones o programas que le permitan ejecutar eficientemente las tareas, por el contrario quienes han optado por destinar capital a tecnologías de la información, mostraron considerables conocimientos acerca de estas herramientas, los que además aseguraron, que en base a los beneficios que han obtenido, los cuales han sido notorios, como la optimización de recursos financieros y materiales, seguirán implementando equipos tecnológicos, sumando la guía de personal especializado, para así obtener mayor provecho de aquellas herramientas. Con dichos resultados se acepta nuestra primera hipótesis específica, donde se establece que las tecnologías de la información poseen beneficios que contribuyen al desarrollo organizacional, adicionalmente se mantiene relación con el autor antes mencionado.

En el libro "La cultura organizacional: eje de acción en la gestión humana", escrito en el 2016 por Llanos Encalada Mónica, Pacheco Rodríguez Miryam, Romero Vélez Eva, Coello Arrata Fabiola, \& Armas Ortega Yadira, en el capítulo II, concluyen que, al implementar una cultura organizacional de calidad y calidez, se requiere del involucramiento de todos los actores de las organizaciones, cambiar hábitos requiere de una práctica permanente, sistemática en la cual el seguimiento y retroalimentación debe ser permanente. El cambio de cultura organizacional, por lo tanto, debe apuntar a una fase de concienciación y adiestramiento del personal que apunte a la participación activa de cada miembro de la empresa, logrando su empoderamiento en el rol que desempeñan y desarrollando una cultura que les sea de beneficio personal y laboral (Llanos Encalada et. al, 2016).

El desinterés en utilizar tecnologías de la información y comunicación en procesos internos de las empresas, se asocian a costumbres remotas que poseen los habitantes del cantón, puesto que, el implementar estas herramientas trae consigo un sinnúmero de cambios no solo en materiales físicos, sino también a los conocimientos, pensamientos, valores, creencias, actitudes, de quienes son parte del talento humano de la organización, que en la actualidad se deben considerar como

(C) Universidad Estatal del Sur de Manabí. Jipijapa, Ecuador. 
activos intangibles, los cuales dan mayor valor a las empresas de acuerdo a las habilidades y destrezas que estos posean, desarrollando de forma eficiente las actividades administrativas. En base a estas tradiciones, una alta cantidad de administradores no son conscientes aun de las herramientas tecnológicas que están disponibles en la actualidad y que brindan a los emprendedores oportunidades para potencializar su negocio, los cuales son generalmente microempresas, aquellas que se han creado únicamente para el sustento diario de la familia, esto debido a que se tienen ilimitadas necesidades y no cuentan con trabajo o fuentes de ingresos, dejando como única opción el emprendimiento, los que son puestos en marcha sin estudios previos y de forma arbitraria.

En la Universidad Técnica Particular de Loja, en el año 2015, la autora Aveiga Plúa Ruth Lizbeth realizó la una investigación titulada “Implementación del uso de las TIC'S en el desempeño de los negocios aplicadas a las pymes ubicadas en la zona urbana del cantón Jipijapa”, donde manifiesta que, en términos de infraestructura tecnológica se observó que las pequeñas y medianas empresas de la Zona Urbana del Cantón Jipijapa, poseen en su mayor porcentaje telefonía móvil, seguido de computador, telefonía fija, internet y fax. Es decir que se concluye que la telefonía móvil es la infraestructura básica; aunque se evidencia un bajo porcentaje que no tiene computador. Las pymes del Cantón Jipijapa utilizan computadora en el área de la administración, en tanto, que el internet es utilizado en la misma área, el correo electrónico y la telefonía móvil se utiliza en menor porcentaje (Aveiga Plúa, 2015).

Los administradores dedican un bajo porcentaje de las herramientas tecnológicas que tienen a su alcance para promover el desarrollo de la empresa. En esta medida y considerando como actividad administrativa a la organización de las tareas a ejecutar, es estala acción que realiza mayoritariamente por los encargados de estas tareas implementando tecnologías de la información y comunicación, que en este sentido son los ordenadores y celulares mediante aplicaciones como WhatsApp, haciendo uso del internet o llamadas telefónicas, dándole un uso claramente básico a estos equipos, desaprovechando gran cantidad de oportunidades para la potencialización de la empresa. Los resultados de la presente investigación, coinciden sorprendentemente con lo expuesto por la autora antes mencionada, lo que evidencia que son pocas las acciones que los dirigentes realizan para llevar más allá a sus emprendimientos. Por el contrario y haciendo referencia, solo a aquellos negocios que si han usado tecnologías de la información y comunicación en el área administrativa, es donde se ha percibido una mejora en el desarrollo de estas actividades. Esta información nos permite validar la segunda hipótesis específica.

En el cantón Jipijapa, en la actualidad predominan las empresas dedicadas a las actividades terciarias, siendo estas la comercialización de productos y prestación de servicios, en muchas de ellas se han implementado tecnologías de la información y comunicación de forma básica, como el uso de teléfonos móviles con acceso a internet y ordenadores, y en menor medida las organizaciones que realizan actividades de las que obtienen recursos naturales y las que se encargan de dar valor agregado a la materia prima. Estas herramientas son utilizadas generalmente para mantener una comunicación interna, que, si bien es cierto, se considera un aspecto importante mantener comunicado a todo el talento humano, al limitar el uso de estos equipos se pierden numerosos beneficios que le servirían a la empresa para poder potencializarse. 
Se considera, que esto se ve ampliamente relacionado a la cultura que ha venido transcendiendo con el paso de los años en la vida de los jipijapenses, recalcando que en tiempos pasados en Jipijapa se sembraba y cosechaba gran cantidad de café de buena calidad, llegándolo a considerar como "La Sultana del Café". Sin embargo, al llegar la era tecnológica en el siglo XX, y hasta la presente fecha los administradores de las empresas establecidas en el Cantón en su gran mayoría no muestran motivación por destinar capital para la adquisición de tecnologías de la información y comunicación que apoyen las actividades que se realicen dentro de la organización. Pese a esto existen limitadas organizaciones que se han logrado potencializar debido al uso de las TIC's, siendo en su mayoría las dedicadas a las actividades del sector terciaria. Se rechaza la tercera hipótesis específica por la falta de empresas potencializadas en las actividades primarias y secundarias en el cantón Jipijapa. Adicionalmente, dentro de la zona urbana y rural hay otros negocios que se dedican a las diferentes actividades económicas que se mencionaron anteriormente, las cuales se han potencializado significativamente, pero es importante mencionar que ha sido gracias a la implementación de otros factores, los cuales son ajenos a las variables de estudio de la presente investigación.

\section{CONCLUSIONES}

Los beneficios que aporta el uso de las tecnologías de la información en el desarrollo organizacional de las empresas son múltiples, estas herramientas están ampliamente relacionadas a los software, acceso a internet, servicios en línea, equipos físicos, entre otros, los cuales facilitan la colaboración de las personas, permite el intercambio de información, mantiene los datos históricos y actuales de forma ordenada y segura, incrementan la eficiencia de los trabajadores, crean motivación e interés en ellos. Es importante recalcar que, para lograr dichas ventajas y entre otras muchas que ofrecen las TIC's, se tiene que lograr una correcta adaptación de las personas que participan en cada una de las áreas a los cambios tecnológicos que se realicen, llevando a cabo procesos paulatinos de cambios que sean planeados y ejecutados de forma estratégica y adecuados a las necesidades, con el fin de familiarizar a los trabajadores con las herramientas a implementar, esto genera una mejor comunicación, mayor confianza, seguridad y compromiso de todo el talento humano para el cumplimiento de las metas, considerando como pilar fundamental la creación de una nueva cultura organizacional que se emplee con el propósito de inyectar mejores actitudes personales, hábitos adecuados, valores morales, ideologías que fomenten la gestión de conocimiento, innovación constante, formas eficientes de interacción y adquisición de herramientas tecnológicas, que frente a las altas competencias que presentan todo tipo de empresa sean estas públicas, privadas, micros, pequeñas, medianas o grandes, representa capital intelectual para ellas, siendo estos, aspectos con los que pueden disminuir considerablemente el impacto de las otras compañías que se encuentren en el mercado.

Las personas que están encargadas de la administración manejan gran cantidad de información, que es muy importante para la toma de decisiones y además este personal es responsable de que estos datos se mantengan ordenados, actualizados, seguros y que no exista información alterada de ningún tipo, por lo que se considera a estas acciones como un soporte para cualquier proceso

(C) Universidad Estatal del Sur de Manabí. Jipijapa, Ecuador. 
que se desee ejecutar, es por ello, que las tecnologías de la información y comunicación han favorecido al desarrollo de las actividades administrativas, en aquellas empresas del cantón Jipijapa que considerablemente decidieron invertir en determinadas herramientas, para ejecutar acciones como la planeación, organización, dirección, coordinación y control, incorporando a todo el talento humano, ya que brindan la oportunidad tener un registro ordenado de las actividades que se implementaran para el cumplimiento de las metas, llevar un control de las herramientas que se necesitaran para cada acción y así obtener mejores beneficios, mantener una comunicación adecuada entre cada departamento, mejorando a su vez la productividad de los trabajadores, aspecto que se considera primordial para alcanzar el éxito, recalcando que existe una mayoría de empresas que no han obtenido estos beneficios por el bajo nivel de inversión que han realizado.

En el cantón Jipijapa las empresas que se han potencializado por el uso de tecnologías de la información y comunicación son escasas, especialmente aquellas que se dedican a las actividades primarias como la agricultura, ganadería, pesca y las secundarias, aquellas que realizan la transformación de los recursos naturales o materia prima en un producto final, situación que se relaciona en gran medida a la falta de cultura tecnológica que poseen los emprendedores, sin embargo existen determinadas microempresas en el sector terciario que se han beneficiado debido a la adecuada implementación de estas herramientas.

\section{REFERENCIAS BIBLIOGRÁFICAS}

Acevedo Díaz, J. A. (Enero de 2006). MODELOS DE RELACIONES ENTRE CIENCIA Y TECNOLOGÍA: UN ANÁLISIS SOCIAL E HISTÓRICO. Obtenido de http://www.redalyc.org/articulo.oa?id=92030203

Aveiga Plúa, R. L. (2015). Implementación del uso de las TIC's en el desempeño de los negocios aplicadas a las PYMES ubicadas en la Zona Urbana del Cantón Jipijapa 2015. Obtenido de http://dspace.utpl.edu.ec/bitstream/123456789/13644/3/Aveiga_Plua_Ruth_Lizbeth.pdf

Belloch, C. (2002). Las Tecnologías de la Información y Comunicación en el aprendizaje. Obtenido de Departamento de Métodos de Investigación y Diagnóstico en Educación. Universidad de Valencia: https://www.uv.es/bellochc/pedagogia/EVA1.pdf

Cabello, C. R. (13 de Abril de 2018). ¿Qué es un ERP? Tipos, Beneficios, Precios (e-book). Obtenido de https://www.sage.com/es-es/blog/que-es-un-erp-ebook/

Cano Pita, G. E. (1 de Enero de 2018). Las TICs en las empresas: evolución de la tecnología y cambio estructural en las organizaciones. Obtenido de https://dialnet.unirioja.es/descarga/articulo/6313252.pdf

Castañeda Gómez, R. (Noviembre de 2011). “EL PROCESO DE CAMBIO EN LAS ORGANIZACIONES. Obtenido de http://eprints.uanl.mx/2341/1/1080227417.pdf

Chavez Cruz, E., \& Negrete Vargas, M. A. (Octubre de 2012). Competitividad e innovación tecnológica como factores de cambio institucional, retos y oportunidades para México. Obtenido de http://congreso.investiga.fca.unam.mx/docs/xvii/docs/D02.pdf

Cobo Romaní, J. C. (22 de Septiembre de 2009). El concepto de tecnologías de la información. Benchmarking sobre las definiciones de las TIC en la sociedad del conocimiento. Obtenido de https://cmapspublic3.ihmc.us/rid=1MNM63T42-7YHX0S-5XD/zer27-14-cobo.pdf

174 UNESUM-Ciencias. Publicación cuatrimestral. Vol. 4, No. 4 (Septiembre-Diciembre), Año 2020. 
Cortes Méndez, J. A., Páez Páez, J. A., \& Oswaldo Lozano, J. (17 de Mayo de 2016). La aplicación de las TIC en los sistemas de gestión de las PyME del sector portuario. Obtenido de Revista Electrónica Redes de Ingeniería: https://revistas.udistrital.edu.co/ojs/index.php/REDES/article/download/8491/11545/0

Delgado Sánchez, L. S. (2013). FACTORES A DESARROLLAR EN LA COMUNICACIÓN INTERNA ENTRE LOS COORDINADORES DE PROYECTOS Y LOS OPERADORES DEL ÁREA TALLER DE LA EMPRESA TAINME S.A. Obtenido de http://repositorio.ug.edu.ec/bitstream/redug/13658/1/TESIS\%20LESLYE\%20SABRINA\%20DELGADO\%20 S\%C3\%81NCHEZ.pdf

Díaz Escoto, A. S. (2011). Informacion y Sociedad del Conocimiento en America Latina. Obtenido de Biblioteca Universitaria: http://www.redalyc.org/pdf/285/28521141003.pdf

Escamilla Aguirre, M. (14 de Diciembre de 2007). Administración de los recursos humanos. Obtenido de http://catarina.udlap.mx/u_dl_a/tales/documentos/lhr/escamilla_a_m/capitulo2.pdf

Escobar García, A. M. (Octubre de 2014). EL USO DE LAS TICS EN LAS PYMES ECUATORIANAS. Obtenido de http://repositorio.puce.edu.ec/bitstream/handle/22000/6917/7.36.000941.pdf?sequence=4\&isAllowed=y

Escorsa Castells, P., \& Valls Pasola, J. (Septiembre de 2003). Tecnologia e innovacion en la empresa. Obtenido de https://books.google.com.ec/books?hl=es\&lr=\&id=53Uxf8gQtuYC\&oi=fnd\&pg=PA1\&dq=innovaci\%C3\%B 3n+tecnologica+empresa\&ots=Gfi3gJXASl\&sig=-tB7KhAxBs4xNxO3W-

WcBRe6kiI\#v=onepage\&q=innovaci\%C3\%B3n\%20tecnologica\%20empresa\&f=false

Expertos en Competencias Empresariales. (06 de Junio de 2018). 4 beneficios evidentes del desarrollo organizacional. Obtenido de CDG: https://www.cdg.com.mx/4-beneficios-evidentes-del-desarrolloorganizacional/

Fuentes Diaz, A. E. (2012). LA CULTURA ORGANIZACIONAL: FACTOR CLAVE DE ÉXITO EN LAS EMPRESAS DEL SIGLO XXI. Obtenido de http://190.242.62.234:8080/jspui/bitstream/11227/1856/1/381\%20ENSAYO\%20-

\%20LA\%20CULTURA\%20ORGANIZACIONAL\%20FACTOR\%20CLAVE\%20DE\%20\%C3\%89XITO\%2 0EN\%20LAS\%20EMPRESAS\%20DEL\%20SIGLO\%20XXI.pdf

Garzón Castrillón, M. A. (2005). El desarrollo organizacional y el cambio planeado. Obtenido de Universidad del Rosario: https://books.google.com.ec/books?hl=es\&lr=\&id=RDFJifNWNMC\&oi=fnd\&pg=PA13\&dq=cambio+planificado+definicion\&ots=y_U9p4ha9\&sig=dBeziUOG7QFfhB27VI9Nq8t8PgA\#v=onepage\&q=cambio\%20planificado\%20definicion\&f=f alse

Garzón Castrillón, M. A. (2005). El desarrollo organizacional y el cambio planeado. Obtenido de Universidad del Rosario: https://books.google.es/books?hl=es\&lr=lang_es\&id=RDFJifNWNMC\&oi=fnd\&pg=PA13\&dq=desarrollo+organizacional\&ots=y_T1at2d9f\&sig=JZVb1QzNIZYDifwt5 I7qm-ExD0w\#v=onepage \&q=desarrollo\%20organizacional $\& \mathrm{f}=$ false

Gomez Díaz , C. F., \& Rodriguez Ortiz, J. K. (Enero de 2001). Teorías de la cultura organizacional. Obtenido de https://www.researchgate.net/publication/216737064_Teorias_de_la_cultura_organizacional

González, M., \& Filippi Sánchez, A. C. (2010). Herramienta informática para gestionar conocimiento a nivel organizacional. Obtenido de http://eprints.rclis.org/16868/1/Gonzalez-Filippi.pdf

(C) Universidad Estatal del Sur de Manabí. Jipijapa, Ecuador. 
GR - Consultores de negocios. (25 de Abril de 2014). El desarrollo organizacional. Obtenido de https://administracionconmihaymon.wordpress.com/2014/04/25/el-desarrollo-organizacional/

Guízar Montúfar, R. (2013). Desarrollo organizacional, principios y aplicaciones. Obtenido de Universidad de La Salle Bajío: https://cucjonline.com/biblioteca/files/original/a8a68a7be0b68ac529abc11ad7d2e85f.pdf

Hernández Medina, A. R. (Diciembre de 2016). Aproximación teórica a modelo de cambio planeado de gestión organizacional para la innovación educativa desde la Teoría de la Complejidad y Empowerment. Obtenido de Universidad Pedagógica Experimental Libertador: https://dialnet.unirioja.es/descarga/articulo/6296681.pdf

176 UNESUM-Ciencias. Publicación cuatrimestral. Vol. 4, No. 4 (Septiembre-Diciembre), Año 2020. 